\title{
Automated system for the measurement of airways resistance, lung volumes, and flow-volume loops
}

\author{
PJ CHOWIENCZYK, PJ REES, TJH CLARK \\ From the Respiratory Function Unit, Guy's Hospital, London
}

ABSTRACT Using a digital computer and body plethysmograph measurements of airways resistance, lung volumes, and flow-volume loops may be obtained from a single manoeuvre performed by the patient. All the measurements together with a display of the flow-volume loop are displayed within 10 seconds of the patient completing the manoeuvre. The system appears to offer considerable ad vantages in speed, objectivity, and reproducibility when compared with conventional methods.

Automated systems for measuring forced expiratory volume and flow are now commonplace in many laboratories. Such systems provide accurate measurements of indices such as forced expiratory volume in one second $\left(\mathrm{FEV}_{1}\right)$, forced vital capacity (FVC), flow at $50 \%$ and $25 \%$ of the vital capacity, and mean transit time (MTT). In contrast measurements of airways resistance and absolute lung volumes, while in common use, are normally derived manually by body plethysmography. These manual measurements are often subject to large intra-subject variability ${ }^{2}$ and, particularly with regard to measurements of airways resistance, exhibit large inter-observer errors. ${ }^{3}$

We have developed an automated plethysmograph system which allows measurements of airways resistance, lung volumes, and forced expiratory flowvolume loops to be obtained from a single manoeuvre performed by the patient. The algorithms used to calculate airways resistance and lung volumes form the most important feature of the system as they allow objective measurements to be made which are more reproducible than those which can be obtained manually.

We describe here our system and present a comparison of plethysmographic measurements determined by conventional methods and by our system.

\section{Methods}

The hardware consists of a constant volume plethysmograph and a digital computer. Two Fleisch pneumotachographs connected in series are used to

Address for reprint requests: Mr PJ Chowienczyk, Respiratory Function Unit, Second Floor New Guy's Hou'se, Guy's Hospital, London Bridge, London SE1 9RT. measure flow at the mouth. One (a no 1 ) is used for low flows during the measurement of airways resistance and the other (a no 4) for high maximal expiratory flows. Differential pressure transducers are used to measure the pressures across the pneumotachographs, pressure in the plethysmograph, and pressure at the mouth. The signals from the no 1 Fleisch pneumotachograph, the plethysmograph pressure transducer and the mouth pressure transducer have a matched frequency response which is flat to 10 hertz $(\mathrm{Hz})$. The plethysmograph has a mouth shutter and venting shutter. Both shutters are controlled from the computer by relays. The pressure transducer signals enter the computer via a 10-bit four-channel analogue to digital converter.

The computer used was a Data General Nova 2/10 mini-computer with a cartridge disk unit (Data General Corporation, Southboro, Massachusetts). The software has, however, been recoded to run on a Research Machines $380 \mathrm{Z}$ microcomputer (Research Machines Ltd, Oxford, England). Peripheral computer equipment includes a high resolution graphics terminal and hard copy unit (Tektronix 4010-1, Tektronix Inc, Beaverton, Oregon, USA).

The manoeuvre performed by the patient which $\stackrel{\sim}{\circ}$ allows all the measurements to be determined may, for descriptive purposes, be divided into three distinct stages.

The first stage consists of a period of rapid shallow breathing or panting at an approximate rate of $120 \stackrel{\circ}{C}$ breaths per minute for a period of two seconds. Plethysmograph pressure and flow are sampled. This stage provides the data from which airways resistance (Raw) may be obtained.

The second stage consists of a further period of panting for about two seconds against a closed $\stackrel{\varnothing}{\varnothing}$ 
mouth-shutter. Mouth pressure and plethysmograph pressure are sampled and used to calculate the thoracic gas volume $(\mathrm{Vtg})$ at this point. During this time flow is also sampled. This allows a flow baseline to be established from which flow may subsequently be integrated to obtain volume.

In the third, final stage, the mouth-shutter opens and the patient inspires to total lung capacity (TLC) and then expires forcibly to residual lung volume (RV). Flow is sampled (from the no 4 Fleisch head) and simultaneously integrated to give volume. This stage allows the lung volumes TLC and RV to be determined from the thoracic gas volume measured during the second stage. The flow-volume loop and indices of the forced expiration are also determined. The inspiratory part of the flow-volume loop may also be recorded if required.

The method by which airways resistance is calculated from data sampled during the first stage of the manoeuvre is described fully elsewhere. ${ }^{5}$ The value of resistance obtained is the average of inspiratory and expiratory resistance. In the appendix we describe the algorithm used for the calculation.

During the second stage of the manoeuvre thoracic gas volume is calculated from the ratio of changes in mouth pressure to those of plethysmograph pressure (the $\triangle \mathbf{P m} / \triangle \mathbf{P p}$ ratio) using the classical method of DuBois. ${ }^{6}$ When thoracic gas volume is determined manually this ratio is taken as the slope of a "best fit" line drawn through an X-Y plot of mouth and plethysmograph pressure. A natural ex- tension of this method to a computerised system is to fit a regression line to digitised mouth and plethysmograph pressure signals. Sykes et al have made use of such a method. ${ }^{7}$ This method will only work, however, if the patient performs the panting manoeuvre correctly. If the patient stops panting or shuts his glottis data will be obtained for which the $\triangle \mathbf{P m} / \triangle \mathbf{P p}$ ratio will give an incorrect value for the thoracic gas volume. During occurrences such as these the mouth pressure signal will remain near stationary in time, as shown in fig 1 , and a large number of "invalid" data points will be obtained which bias the slope of a simple regression line. We have found that by using only data points for which the rate of change of mouth pressure is greater than a certain lower limit we can avoid this bias. Details of the algorithm are given in the appendix. Also described in the appendix are the algorithms for calculating the lung volumes and indices of the forced expiration during the third stage of the manoeuvre.

The $\mathrm{Pp}$ and $\mathrm{Pm}$ channels are calibrated by the introduction of known volumes and pressures respectively. The two flow channels are calibrated by introducing known volumes and comparing these with volumes obtained by integrating the flow signal. After the computer has completed the integration, flow and volume are displayed as an X-Y plot. This allows the operator to check that the volume was introduced at a rate within the limits of linearity of the Fleisch head.
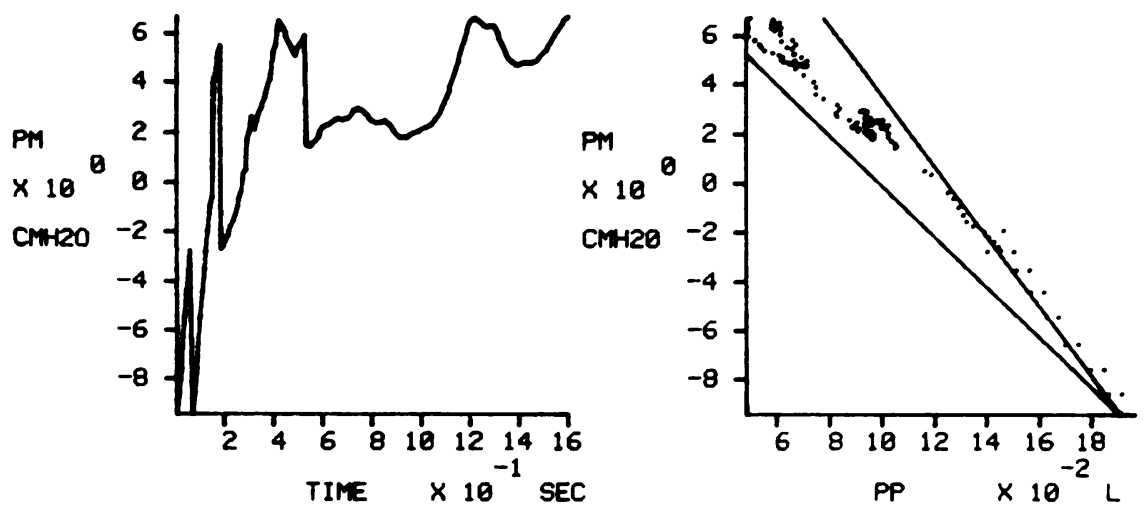

Fig 1 The behaviour of mouth pressure Pm as a function of time and as a function of plethysmograph pressure Pp during panting against a closed mouth shutter. When mouth pressure is stationary in time the patient has either stopped panting or shut his glottis. These occurrences give rise to the "clusters" of data points on the X-Y plot. These clusters of points do not have a welldefined slope and will bias the slope of a simple regression line. The lower line on the X-Y plot is such a regression line, shown slightly displaced so that it does not obscure the data. The upper line is a line for which the slope has been calculated using data points for which the rate of change of mouth pressure is above a threshold value. This line is parallel to the straight segments of the $X-Y$ plot and will therefore give a more accurate value for the thoracic gas volume. 
Operation of the system is extremely simple. The patient sits in the plethysmograph and is instructed on how to perform the manoeuvre. The door of the plethysmograph is then sealed. The plethysmograph is periodically vented until the increase in plethysmograph pressure caused by thermal radiation from the patient is minimal. This usually takes about two minutes. A "start" button on the computer is pressed and a signalling light near the plethysmograph used to indicate to the patient to start the manoeuvre.

Ten seconds after completing the manoeuvre a print-out of results may be obtained together with a display of the flow-volume loop. The signals sampled during the first and second stages of the manoeuvre may also be displayed. The pressure and flow signals corresponding to the airways resistance measurement are displayed as an X-Y plot or "airways resistance loop". When airways resistance is determined manually a straight line is fitted by eye to this loop. Resistance is calculated from the slope of this line. On the computer display a line is also drawn which has a slope corresponding to the value of resistance calculated by the computer program. The signals sampled during the determination of thoracic gas volume are also displayed as an X-Y plot together with a "computer fitted" line having a slope giving the thoracic gas volume determined by the program. All the results and displays are stored on a disk file and may be recalled at a later date.

The computerised method of determining airways resistance has been compared with the conventional method and has been found to correlate well. ${ }^{4}$ In order to verify the algorithm for determining thoracic gas volume a comparison was made with the conventional manual method. Graphical displays of the X-Y plot of plethysmograph pressure and mouth pressure were obtained (without the computer fitted lines). Lines were fitted to these plots by eye and used to obtain manual estimates of thoracic gas volume which could be used to calculate total lung capacity from the inspired volume relative to $\mathrm{Vtg}$ measured by the computer during the third stage of the manoeuvre. These "manual" TLC values could then be compared with the values obtained by the computer. This comparison was made for 10 patients with varying degrees of airways obstruction and eight normal subjects. Each subject was asked to perform the manoeuvre six times.

\section{Results and discussion}

The standard output obtainable from the system is illustrated in fig 2 . We routinely perform measure-

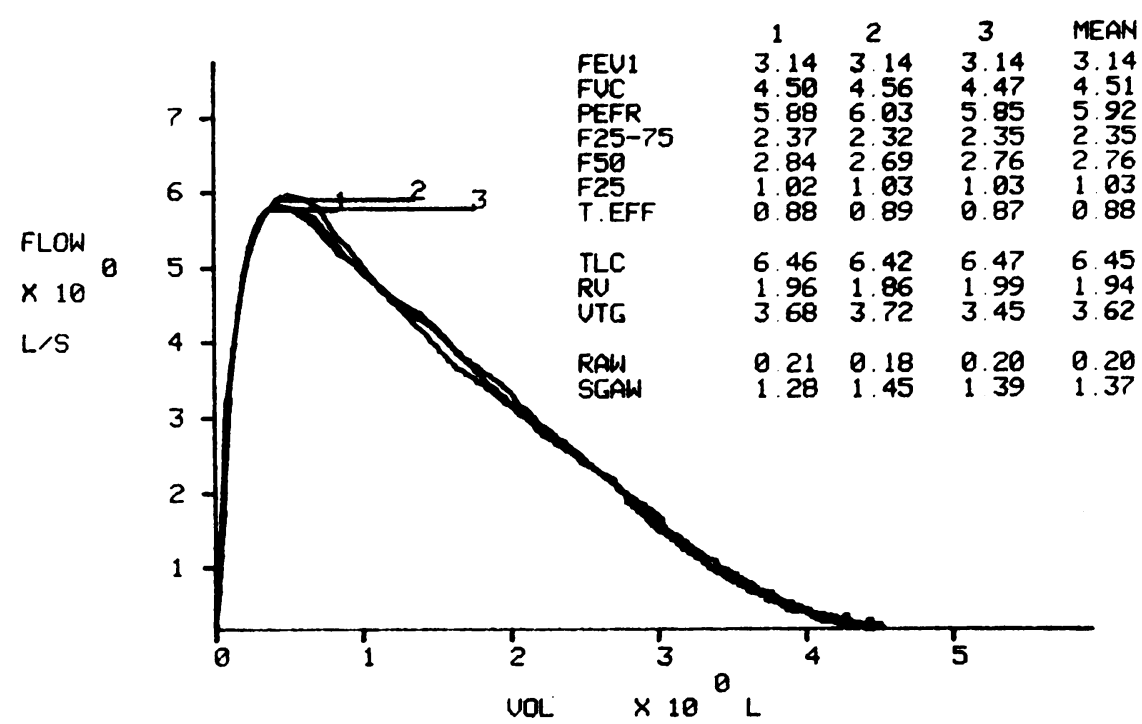

Fig 2 The hard copy output by the system after three manoeuvres have been performed by the patient. The flow-volume loops are shown together with measurements derived during the first, second, and third stages of the manoeuvre: FEV ; forced expiratory volume in one second, FVC; forced vital capacity, PEFR; peak expiratory flow rate, F25-75; mean flow from $25 \%$ of the vital capacity to $75 \%$, F50; flow at 50\% of the vital capacity, F25; flow at $25 \%$ of the vital capacity, T.EFF; effective time constant (or mean transit time), TLC; total lung capacity, $R V$; residual lung volume, VTG; thoracic gas volume, $R A W$; airways resistance, sGaw; specific airways conductance. 


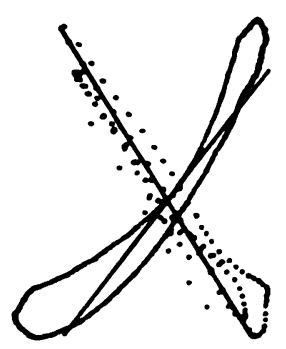

$\begin{array}{ll}\text { RAW } & 0.39 \\ \text { UTG } & 6.28 \\ \text { SGAW } & 0.41\end{array}$

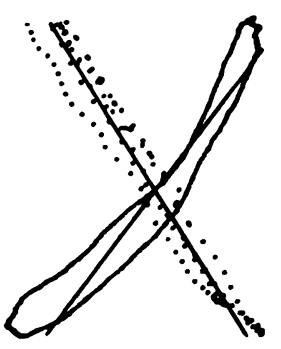

อ. 38

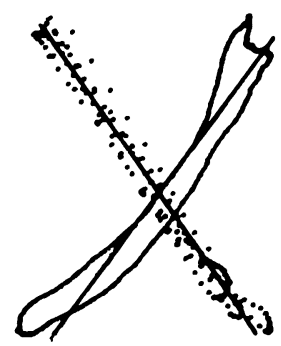

0. 39

5.77
0. 39

5. 94

0. 44

Fig 3 The $X-Y$ displays that may be generated by the computer system. In this case the displays were obtained from a mild asthmatic. The solid loops are the airways resistance pressure-flow loops with pressure on the $X$-axis and flow on the $Y$-axis. The dotted $X$-Y plots are the thoracic gas volume plots with plethysmograph pressure on the $X$-axis and mouth pressure on the $Y$-axis. The lines drawn through the plots are the "computer fitted" lines having slopes which give the results obtained by the computer program.

ments in groups of three and the flow-volume loops are displayed superimposed so as to judge reproducibility. Figure 3 illustrates the $X-Y$ airways resistance and thoracic gas volume plots together with the computer fitted lines that may be displayed by the system. The results of the comparison of the manual and computer methods of measuring total lung capacity are shown in fig 4 . The regression line

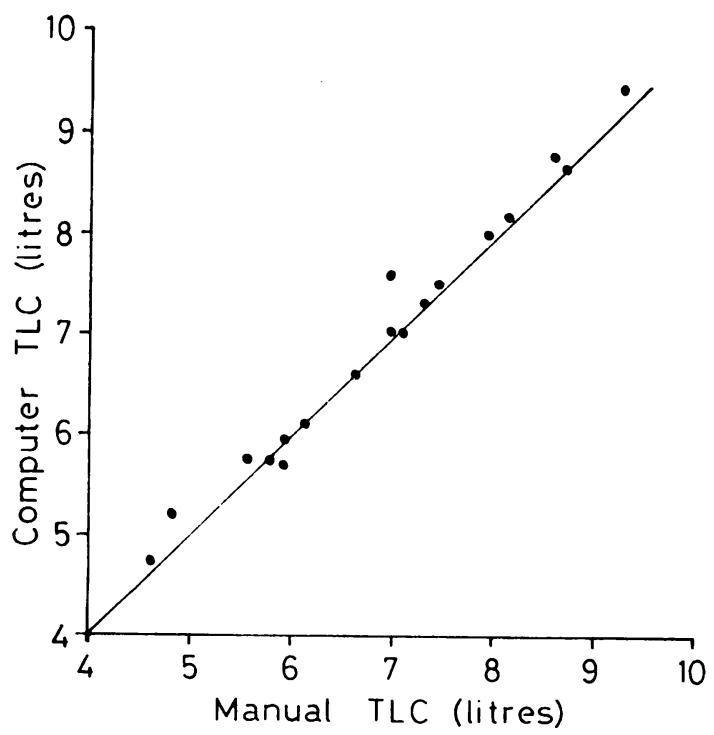

Fig 4 Comparison of mean values of total lung capacity obtained by the computer system and those obtained from manual estimates of thoracic gas volume. The solid line is the line of identity. of computer values of TLC on manual values has a slope of 1.0 and an intercept of 0.09 . These values are not significantly different from unity and zero respectively $(p<0.05)$. The mean intra-subject standard deviation of total lung capacity is $180 \mathrm{ml}$ for the computer determinations and $305 \mathrm{ml}$ for the manual estimates. This difference is not significant by paired $t$ test $(t=1 \cdot 81)$. It should be remembered, however, that manual estimates of thoracic gas volume and hence total lung capacity vary with the observer. ${ }^{3}$ This source of variation is eliminated in the computer system.

Measurements of airways resistance also exhibit large intra-subject and inter-observer variability. ${ }^{2} 3$ In a separate study we have found that our system provides measurements of specific airways conductance, derived from airways resistance and thoracic gas volume, which have a coefficient of variation in the order of $10 \%{ }^{4}$ This is significantly less than measurements made by manual methods.

We have found the speed and ease of operation of the system to be extremely valuable when studying the effects of various bronchodilator treatments on asthmatic patients. Three sets of manoeuvres and measurements may be made in less than five minutes. To obtain these measurements by conventional techniques would require nine manoeuvres to be performed (three flow-volume loops, three airways resistance manoeuvres, and three lung volume manoeuvres). The plethysmographic measurements would then have to be calculated from lines fitted by eye to the $\mathrm{X}-\mathrm{Y}$ plots. This is time-consuming and produces errors. Furthermore the results would not be available for inspection during the course of the 
study, a factor of importance in challenge studies.

The system allows measurements of airways resistance to be performed without the maximal inspiration and expiration (that is without the third stage of the manoeuvre). We have used this mode of operation extensively to investigate the acute effects of irritants such as cigarette smoke. ${ }^{8}$

\section{References}

${ }^{1}$ Higenbottam T, Clark TJH. A method for standardising airways resistance for variations in lung volume. Clin Sci 1979;57:397-400.

${ }^{2}$ Lord PW, Brooks AGF. A comparison of manual and automated methods of measuring airway resistance and thoracic gas volume. Thorax 1977;32:60-6.

${ }^{3}$ Lord PW, Brooks AGF, Edwards JM. Variation between observers in the estimation of airways resistance and thoracic gas volume. Thorax 1977;32:67-71.

${ }^{4}$ Chowienczyk PJ, Rees PJ, Payne J, Clark TJH. A new method for the computer assisted determination of airways resistance. $J$ Appl Physiol 1981;50:672-8.

${ }^{5}$ Chowienczyk PJ, Rees PJ, Payne J, Clark TJH. A new method for the computer-assisted determination of airways resistance. Clin Sci 1980;58:25p.

${ }^{6}$ DuBois AB, Botelho SY, Bedell GN, Marshall R, Comroe JH Jr. A rapid plethysmographic method for measuring thoracic gas volume. J Clin Invest 1956;35:322-6.

${ }^{7}$ Sykes TW, Haynes RL, McFadden ER Jr. On line determinations of lung volumes by plethysmography and digital computer. Am Rev Respir Dis 1977;115:581-5.

${ }^{8}$ Rees PJ, Chowienczyk PJ, Clark TJH. Immediate response to inhaled lung irritants in man. Clin Sci 1980;58:5p.

\section{Appendix}

We describe here the algorithms implemented by the computer software for calculating airways resistance, lung volumes, and the indices of the forced expiration.

\section{AIR WAYS RESISTANCE}

During the first stage of the manoeuvre the subject starts to pant at a frequency of approximately $2 \mathrm{~Hz}$. The instant at which the flow signal crosses the flow baseline from expiratory flow to inspiratory flow is first identified. Plethysmograph pressure $(\mathrm{Pp})$ and flow $(\dot{\mathrm{V}})$ are sampled and digitised at a rate of $100 \mathrm{~Hz}$ and stored in memory until the flow signal recrosses the baseline in the same direction-that is, after one cycle of panting has been completed. The number of data samples collected during this time is then used to check the frequency of the panting. If this is between 1 and $3 \mathrm{~Hz}$ the program proceeds to the thoracic gas volume determination (second stage of the manoeuvre). If not the memory containing the pressure and flow signals is overwritten, the process repeated and a second cycle stored. If, after five cycles, a cycle of frequency in the range $1-3 \mathrm{~Hz}$ is not obtained the program issues an appropriate error message and the patient is instructed to re-start the manoeuvre.

Calculation of airways resistance from stored pressure and flow signals takes place after the patient has completed the manoeuvre. Resistance is determined from the amplitudes and phase relation of sine waves fitted to one cycle of the pressure and flow waveforms according to the formulae:

$$
\text { Raw }=\overline{\text { S. }} \frac{\text { PM.cos } \theta}{\dot{\mathrm{VM}} \cdot 10.2}
$$

where $\bar{S}$ is the value of the $\triangle P m / \triangle P p$ ratio determined in the second stage of the manoeuvre, PM the amplitude of the sine wave fitted to the pressure waveform, VM the amplitude of that fitted to the flow waveform, and $\theta$ the phase angle between these sine waves. The units of $\overline{\mathbf{S}}$, $\mathrm{PM}$, and $\dot{\mathrm{V}} \mathrm{M}$ are, respectively, $\mathrm{cm} \mathrm{H}_{2} \mathrm{O}^{-1} \mathrm{l}^{-1}, 1$, and $\mathrm{l} / \mathrm{s}$. The units of Raw are kPa.l. ${ }^{-1} \mathrm{~s}$. PM, VM, and $\theta$ are computed using the principle of least squares. If the n'th point to be digitised on the pressure waveform is $\mathrm{Pn}$ and that on the flow waveform $\mathrm{V} n$ and $\mathrm{N}$ points are obtained in total the formulae are:

$$
\begin{aligned}
\mathrm{PM} & =\sqrt{\mathrm{a}^{2}+\mathrm{b}^{2}} \\
\dot{\mathrm{V} M} & =\sqrt{\mathrm{c}^{2}+\mathrm{d}^{2}} \\
\theta & =\arctan (\mathrm{b} / \mathrm{a})-\arctan (\mathrm{d} / \mathrm{c})
\end{aligned}
$$

where

$$
\begin{array}{ll}
\mathrm{a}=\frac{2}{\mathrm{~N}} \sum_{\mathrm{n}=1}^{\mathrm{N}} \operatorname{Pn} \cdot \cos (\mathrm{wn}) & \mathrm{b}=\frac{2}{\mathrm{~N}} \sum_{\mathrm{n}=1}^{\mathrm{N}} \mathrm{Pn} \cdot \sin (\mathrm{wn}) \\
\mathrm{c}=\frac{2}{\mathrm{~N}} \sum_{\mathrm{n}=1}^{\mathrm{N}} \dot{\mathrm{V}} \mathrm{n} \cdot \cos (\mathrm{wn}) & \mathrm{d}=\frac{2}{\mathrm{~N}} \sum_{\mathrm{n}=1}^{\mathrm{N}} \dot{\mathrm{V}} \mathrm{n} \cdot \sin (\mathrm{wn}) \\
\mathrm{w}=2 \pi / \mathrm{N} &
\end{array}
$$

\section{THORACIC GAS VOLUME}

Thoracic gas volume is computed during the second stage of the manoeuvre while the patient pants against a closed mouthpiece. Mouth pressure and plethysmograph signals are digitised at a rate of $100 \mathrm{~Hz}$. The approximate rate of change, $\triangle \mathbf{P m}$, of mouth pressure is monitored by subtracting each digitised mouth pressure point from the previous one. If the magnitude of $\triangle \mathrm{Pm}$ is greater than a threshold value $\mathrm{Pl}$ the digitised pressure signals are stored in memory. If the magnitude of $\triangle \mathrm{Pm}$ is less than $\mathrm{Pl}$ and provided at least five data points have been sampled data acquisition is momentarily terminated and a regression line fitted to the digitised $\mathrm{Pm}$ and $\mathrm{Pp}$ signals stored in memory. The slope $\mathbf{S}$ of the regression line is stored. Data acquisition is then re-started and the process repeated until a total of $\mathbf{N}$ data points have been sampled. This method effectively divides the set of $\mathrm{N}$ sampled points into subsets for which the rate of change of mouth pressure $(\triangle \mathrm{Pm})$ is greater than $\mathrm{Pl}$. The average $\overline{\mathrm{S} \text { of the values }}$ $\mathbf{S}$ of the slopes of the regression lines for each subset is used to calculate thoracic gas volume (Vtg). Since $\triangle \mathrm{Pm}$ falls to zero during changes from inspiratory to expiratory effort against the shutter (and vice versa) the number of subsets obtained is usually twice the number of panting cycles. If the number of subsets is less than three or if the standard deviation of the values $S$ (in terms of the thoracic gas volume calculated from $\mathbf{S}$ ) is greater than one litre the program issues an error message. The formula used to calculate $\mathrm{Vtg}$ from $\mathrm{S}$ is:

$\mathrm{Vtg}=1 \cdot 36 .(1-\mathrm{W} /(1 \cdot 07 . \mathrm{Vp})) .(\mathrm{Patm}-47) / \overline{\mathrm{S}}$

Where Patm is the atmospheric pressure (in $\mathrm{mm}$ of mercury), $\mathrm{W}$ the weight of the patient (in $\mathrm{Kg}$ ), and $\mathrm{Vp}$ 
the volume of the plethysmograph (in 1). The factor $(1-W /(1 \cdot 07 . V p))$ is used to correct for the volume occupied in the plethysmograph by the patient. The values of $\mathrm{N}$ and $\mathrm{Pl}$ normally used are $\mathrm{N}=200$ and $\mathrm{PI}=0.5$ cmH20.

TOTAL LUNG CAPACITY, RESIDUAL LUNG VOLUME, AND INDICES OF THE FORCED EXPIRATION

During the third stage of the manoeuvre volume is obtained by digital integration of flow. The problem normally associated with flow integration is that of drift which arises when the value of the flow signal corresponding to zero flow (the flow baseline) is unknown. In the second stage of the manoeuvre the mouth shutter is closed and flow is zero. At this time the flow signal is digitised at $100 \mathrm{~Hz}$ and the mean value of these samples used as the flow baseline from which to integrate flow in the third stage of the manoeuvre. This eliminates problems of drift. In the third stage the patient inspires to total lung capacity (TLC) and then expires forcibly to residual lung volume (RV). During this time the flow signal is digitised at $100 \mathrm{~Hz}$ and integrated to give values for the lung volume (relative to the thoracic gas volume determined during the second stage). The digitised flow samples and corresponding volume values are stored in memory until the expired volume is greater than 0.5 litres and flow has been between the values $F 1$ and $F 2$ for two seconds, at which time expiration is assumed to have finished. If this does not occur within 20 seconds of the start of the third stage the program issues an error message and the patient is instructed to re-start the manoeuvre.

The array of values of volume in memory is then scanned to find the maximum. Total lung capacity is calculated by first correcting this inspired volume to body temperature and pressure and then adding it to the thoracic gas volume determined during the second stage. The point in the stored flow and volume arrays corresponding to the onset of the forced expiration is taken as the point at which volume is equal to the maximal inspired volume and flow greater than a threshold value F3 in the expiratory direction. Having identified the start of the forced expiration the vital capacity and indices of the forced expiration may be computed from the flow and volume arrays. The threshold values F1, F2, and F3 used are: $\mathrm{F} 1=0.04 \mathrm{l} / \mathrm{s}, \mathrm{F} 2=-0.04 \mathrm{l} / \mathrm{s}, \mathrm{F} 3=0.2 \mathrm{l} / \mathrm{s}$. 